\title{
Mass-spectrum of Charged Leptons from the Planck Mass
}

\author{
A.A. Kotkov \\ P.N. Lebedev Physical Institute of the Russian Academy of Sciences, Russian Federation
}

Copyright $(2016$ by authors, all rights reserved. Authors agree that this article remains permanently open access under the terms of the Creative Commons Attribution License 4.0 International License

\begin{abstract}
There are three generations of charged leptons - the electron, muon, and tau. Masses of elementary particles are considered as fundamental constants. Modern physics believes these masses could be calculated from more fundamental mass scale, e.g., the Planck mass. Scientists seek for such relationship for many years. However, a relation between mass-spectrum of charged leptons and the Planck mass is still unknown. Here we show a way to derive the mass-spectrum of charged leptons from the Planck mass.
\end{abstract}

Keywords Electron, Muon, Tau-lepton

\section{Introduction}

The Standard Model (SM) is the most important theory in modern physics that was developed as a collaborative effort of scientists around the world [1]. Leptons are an important part of the SM. However, the SM has dozens of free parameters that are not predicted by the theory, e.g., rest masses of leptons.

Charged leptons also known as the electron-like leptons can combine with other particles to form various composite particles such as atoms. There are three generations of charged leptons: the electron, muon, and tau ( $\tau$ lepton) with their antiparticles. In the SM, each lepton starts out with no intrinsic mass. The charged leptons obtain an effective mass through interaction with the Higgs field, a fundamental field of crucial importance to particle physics theory.

Masses of elementary particles (including charged leptons) are considered as fundamental constants [2, 3]. From the standpoint of modern physics, however, it would be better if these masses be derived from a more fundamental mass (energy) scale, e.g., from the Planck mass [4].

There are several phenomenological formulas for the mass-spectrum of charged leptons at present. However, a relation between the mass-spectrum of charged leptons and the Planck mass is still unknown. Here this work shows a way to derive the mass-spectrum of charged leptons from the
Planck mass.

\section{Historical Background}

In 1952, Nambu [5] proposed a unit of mass $M_{N}=m_{e} / \alpha \approx$ $137 m_{e}$, where $m_{e}$ is the electron mass and $\alpha$ is the fine-structure constant. He suggested that the mass of a particle can be written as $m(N) \approx N M_{N}$, where $N$ is "mass number," which can be either integer $\{0,1,2 \ldots\}$ or half-integer $\{1 / 2,3 / 2,5 / 2 \ldots\}$. For example, $N$ is equal to 2 for the pion ( $\pi$ meson) and $N=3 / 2$ for the muon $m_{\mu}$

$$
m_{\mu} \approx \frac{3}{2} \frac{m_{e}}{\alpha} .
$$

At the time only eight massive particles were known with poor accuracy of their masses. The development of experimental methods resulted in the increasing accuracy of mass measurement, and the number of massive particles increased dramatically. Nevertheless, the hypothesis of "mass numbers" was further developed in [6].

In 1979, Barut [7, 8] considered the muon and tau as excited states (generations) of the electron. Barut suggested that the energy of the system, which consists of elementary electric charge and intrinsic angular momentum (spin of 1/2) was proportional to the fourth power of the new quantum number $B$, which was equal to generation number: $B=0$ for the electron, $B=1$ for the muon and $B=2$ for the tau.

The Barut's formula has a relatively simple form

$$
m(B)=m_{e}\left(1+\frac{3}{2 \alpha} \sum_{k=0}^{B} k^{4}\right) \text {. }
$$

Equation (2) gives a good agreement with experimental data, as a deviation of the calculated masses from the measured values [3] is about $0.1 \%$ for the muon and $0.5 \%$ for the tau. Due to its simplicity and accuracy, the Barut's formula has attracted the attention of many researchers (see e.g. $[9,10])$.

In 1982, Koide [11, 12] found out a correlation between the masses of three generations of charged leptons 


$$
\frac{3}{2} \frac{\left(m_{1}+m_{2}+m_{3}\right)}{\left(\sqrt{m_{1}}+\sqrt{m_{2}}+\sqrt{m_{3}}\right)^{2}}=1 .
$$

Solving the equation (3), Koide received the tau-lepton mass equal to $1.77697 \mathrm{GeV} / c^{2}$. At the time this prediction was more than two standard deviations away from the measured value $1.7842(32) \mathrm{GeV} / \mathrm{c}^{2}$ [13], hereinafter the digits in parentheses are the uncertainties in the last figures (the standard deviation). This prediction stimulated to undertake more accurate measurement of the tau lepton mass and now the tau-lepton mass is equal to $m_{\tau}=1.77682(16)$ $\mathrm{MeV} / \mathrm{c}^{2}$ [3]. When substituting the Committee on Data for Science and Technology (CODATA) recommended masses of the electron, muon and tau [3] into (3), one can obtain the value of $0.99999(21)$. Equation (3) has no theoretical explanation and may be coincidental. Nevertheless, there are papers (see e.g. [14] and references cited therein) studying gauge symmetry, which could lead to (3) for leptons and quarks.

In 1984, Lipmanov [15] suggested the existence of a discrete equation, which described the mass-spectrum of six generations of leptons. To find the equation, the principle of scale invariance has been used, i.e. the desired mass-spectrum must be invariant to the level of mass conversion. Thus, the equation includes only the relationship between masses of leptons. By making a few assumptions (a simplicity of the equation is one of them), he obtained the exponential formula for the mass-spectrum of leptons, which can be represented as follows

$$
m(L)=M_{0} \exp \left(C_{1} L+C_{2} 2^{-L}\right) .
$$

Three constants $M_{0}, C_{1}$ and $C_{2}$ can be calculated by substituting into (4) the measured masses of three generations of leptons (the electron, muon, and tau) that corresponds to three successive numbers $L$. Note equation (4) can fit any three positive values. However, the predictive power of (4) is small. For example, equation (4) gives the mass of the hypothetical lepton of fourth generation about $8.5 \mathrm{GeV} / c^{2}$. However, experimental data exclude the existence of the fourth charged lepton with a mass less than $100 \mathrm{GeV} / c^{2}$ [2]. Perhaps for these reasons, equation (4) remained little known.

Thus, the relation between the mass-spectrum of charged leptons and the Planck mass is still unknown.

\section{Novel Formula}

In order to derive a novel phenomenological formula for the mass spectrum of the charged leptons we drew on the scale invariance from [15]. As a simple function describing the mass spectrum, we took an exponential function, which worked well in (4). When analyzing relationship between masses of the charged leptons, we found out that the logarithm of this relation can be described as the difference between reversed integers $K_{n}$. It means that masses of charged leptons can be presented as follows

$$
m(n) \sim \exp \left(-\frac{A}{K_{n}}\right),
$$

where $A$ is the dimensionless constant; $n$ is the lepton generation number $n=1,2,3$; $K_{n}$ is the sequence of integer numbers, which can be written as

$$
K_{n}=1+\prod_{k=0}^{n}(1+k !)
$$

where $0 !=1$. This sequence $K_{n}$ for $n=1,2,3$ can be calculated with recurrence relation

$$
2 K_{n}=K_{n-1}^{2}+1
$$

where $K_{0}=3$. In addition, the sequence $K_{n}$ can be derived by the following expression

$$
K_{n}=1+4 \prod_{k=1}^{n<4}\left(2^{k}-1\right) .
$$

We suppose that the sequence $K_{n}$ is limited by dimension of the space-time $n<4$. It will be discussed later.

The constant $A$ can be calculated from measured masses of the electron $m(1)=m_{e}$ and the muon $m(2)=m_{\mu}[3]$

$$
A=\ln \left(\frac{m_{e}}{m_{\mu}}\right) \frac{K_{1} K_{2}}{K_{1}-K_{2}}=43.319239 \text { 92(18). }
$$

Now let us consider the relationship between the masses of charged leptons and the Planck mass $M_{P}=(\hbar c / G)^{1 / 2}$, where $G$ is the gravitational constant. The Planck mass is many orders of magnitude higher than the masses of leptons. Nevertheless, we found out a quite simple relationship between them. The novel formula for the mass-spectrum of charged leptons is

$$
m(n)=M_{A} \exp \left[-A\left(1+\frac{1}{K_{n}}\right)\right],
$$

where $M_{A}$ is the scaling factor, which depends on the fundamental constants only

$$
M_{A}=\frac{\pi}{2} M_{P} e^{\alpha / 2}=\sqrt{\frac{\pi h c}{8 G} e^{\alpha}} .
$$

The scaling factor $M_{A}$ can be derived from (11). However, higher accuracy gives the following expression

$$
\begin{aligned}
& M_{A}=m_{e} \exp \left[A\left(1+\frac{1}{K_{1}}\right)\right]= \\
& =1.92481162 \text { (43) } 10^{19} \mathrm{GeV} / \mathrm{c}^{2} .
\end{aligned}
$$




\section{Discussion}

In the SM, the mechanism of electroweak symmetry breaking is associated with a complex doublet scalar field that is the Higgs field. The presence of the Higgs field, now believed to be confirmed [16], explains why some particles have mass. In the SM, any field connected with gravity, which carries an intrinsic scale close to the Planck mass. In the SM, the Higgs boson is a quantum excitation of the Higgs field and the Higgs mass should be very close to the Planck mass [17].

The Planck mass can be derived approximately by setting it as the mass whose Compton wavelength and Schwarzschild radius are approximately equal [18]. The Compton wavelength is equal to $\lambda_{\mathrm{C}}=h /\left(M_{X} c\right)$ and the Schwarzschild radius is $R_{\mathrm{S}}=2 G M_{x} / c^{2}$. Setting them equal $R_{\mathrm{S}}=x \lambda_{\mathrm{C}}$, where $x$ is a factor, which is a relation between Schwarzschild radius and Compton wavelength, we obtain

$$
M_{x}=\sqrt{\frac{x h c}{2 G}} .
$$

If we compare $M_{x}$ with the Planck mass $M_{P}$, the factor is equal to $x=\pi>1$ that is Schwarzschild radius is higher than Compton wavelength for hypothetical particle with the Planck mass. When comparing (11) and (13), we can derive the factor equal to $x=e^{\alpha} \pi / 4 \approx 0.79<1$ that is Schwarzschild radius is lower than Compton wavelength for hypothetical particle with the mass $M_{A}$ and its dynamics would be strongly affected by quantum gravity.

The measured Higgs mass of $125 \mathrm{GeV} / \mathrm{c}^{2}$ [16] is much smaller than the Planck mass. The existence of light Higgs limits theoretical models. The review [19] discusses electroweak symmetry breaking from strong dynamics, where the strong dynamics produces a light composite Higgs doublet. The SO (5)/SO (4) is the minimal coset containing 'custodial' $\mathrm{SO}(4) \cong \mathrm{SU}(2)_{\mathrm{L}} \times \mathrm{SU}(2)_{\mathrm{R}}$ symmetry that gives rise to a Higgs bi-doublet. Other models with larger cosets that also implement custodial symmetry can be reduced to this one.

Note that SO (4) is the group of rotations about a fixed point in four-dimensional space. Assuming that a fixed orientation has been chosen for 4-dimensional space, 4D rotations may be put into left- and right-rotations.

Let us consider the Higgs field around a lepton as the densest packing of identical spheres (hyper-spheres) in 4D space [20]. The kissing number $N_{k}$ for the packing is equal to 24 [21, 22]. It means that the central sphere touches 24 adjacent spheres (24-cell) the centers of which are located in the boundary of 4-ball (3-sphere). These 24 spheres can be called a shell for the central sphere. Summing up spheres in the shell with the central sphere, we get 25 spheres. If we connect the center of the central sphere with the centers of two adjacent spheres in the shell by two radius vectors, then the angle between the two vectors is equal to $\theta=60^{\circ}$ [21].

The constant $A$ in (9) can be derived approximately by the following expression

$$
A \approx 25 \sqrt{3}=\left(N_{k}+1\right) \tan (\theta) .
$$

This expression has a good accuracy as a deviation of the approximated value (14) from the actual value (9) is less than $0.05 \%$. Then the constant $A$ can be considered as a kind of structure constant, which characterizes the structure of the Higgs field around a lepton.

The sequence $K_{n}$ can be considered as a sum of excitations of the Higgs field. Then the one in (6) and (8) corresponds to a centrally symmetric excitation of the central sphere inside the shell. The fore linear excitations (vibrations) of the central sphere inside the shell generate the electron $K_{1}=1+$ 4. The eight rotations of the central sphere inside the shell generate the muon $\mathrm{K}_{2}=\mathrm{K}_{1}+8$. More complex excitations of the shell (24-cell) along the boundary of 4-ball (3-sphere) generate the tau $\mathrm{K}_{3}=\mathrm{K}_{2}+24 \times 3$.

In table 1 we compare mass-spectra of charged leptons derived with the above formulas. In the first two rows of the table 1 we show the CODATA 2014 recommended values of masses [3]. It is worth noting that the first row represents more accurate masses measured in the unified atomic mass unit (u), which is the standard unit that is used for indicating mass on an atomic or molecular scale (atomic mass). These values were applied to derive a relationship between masses of the electron and muon in (9). The second row represents masses measured in the energetic unit of $\mathrm{MeV} / \mathrm{c}^{2}$. These data were applied to derive masses in equations (1) - (3).

As the first formula for mass-spectrum we consider the Nambu's formula [5]. Equation (1) gives mass of the muon with error less than $0.6 \%$ in comparison with $m_{\mu}$.

The next formula is the Barut's formula [7]. Equation (2) gives the mass of the muon with error about $0.1 \%$ in comparison with $m_{\mu}$ and the mass of the tau with error about $0.5 \%$ in comparison with the CODATA recommended mass $m_{\tau}$.

The Koide's formula (3) gives the mass of the tau with high accuracy within one standard deviation in comparison with CODATA recommended value $m_{\tau}$. 
Table 1. Comparison between mass-spectra of charged leptons

\begin{tabular}{|c|c|c|c|c|}
\hline Quantity & Unit & Electron & Muon & Tau-lepton \\
\hline \multirow{2}{*}{$\begin{array}{l}\text { CODATA recommended } \\
\text { masses [3] }\end{array}$} & $\mathrm{u}$ & $5.48579909070(16) \times 10^{-6}$ & $0.1134289257(25)$ & $1.90749(17)$ \\
\hline & $\mathrm{MeV} / \mathrm{c}^{2}$ & $m_{e}=0.5109989461(31)$ & $m_{\mu}=105.6583745(24)$ & $m_{\tau}=1776.82(16)$ \\
\hline \multirow{3}{*}{$\begin{array}{l}\text { Equation (1) [5] } \\
\text { "mass number" } N \\
\text { masses }\end{array}$} & & & & \\
\hline & & - & $3 / 2$ & \\
\hline & $\mathrm{MeV} / \mathrm{c}^{2}$ & $m_{e}$ & 105.037876 71(66) & - \\
\hline \multirow{3}{*}{$\begin{array}{c}\text { Equation (2) [7] } \\
\text { “new quantum number” } B \\
\text { masses }\end{array}$} & & & & \\
\hline & & 0 & 1 & 2 \\
\hline & $\mathrm{MeV} / \mathrm{c}^{2}$ & $m_{e}$ & 105.548875 65(66) & $1786.154903(11)$ \\
\hline \multirow{2}{*}{$\begin{array}{c}\text { Equation }(3)[11,12] \\
\text { masses }\end{array}$} & & & & \\
\hline & $\mathrm{MeV} / \mathrm{c}^{2}$ & $m_{e}$ & $m_{\mu}$ & $1776.969011(47)$ \\
\hline \multirow{3}{*}{$\begin{array}{c}\text { Equation (10) this work } \\
\text { number of generation } n \\
\text { integer } K_{n} \\
\text { masses }\end{array}$} & & 1 & 2 & 3 \\
\hline & & 5 & 13 & 85 \\
\hline & $\mathrm{MeV} / \mathrm{c}^{2}$ & $0.5109989450(40)$ & $105.6583744(59)$ & $1777.249306(78)$ \\
\hline
\end{tabular}

Equation (10) accurately reproduces the measured masses of the electron and muon. The calculated mass of the tau is slightly greater than the measured mass $m_{\tau}$ [3] in approximately three standard deviations.

All the previous works $[5,7,11,12,15]$ considered the mass of charged leptons as fundamental constants between which there must be some relation. In this work we are trying to understand the origin of these particles.

It is of interest that the gravitational constant $G$ can be calculated with high accuracy from equation (11)

$$
G=\frac{\pi h c}{8 M_{A}^{2}} e^{\alpha}=6.6740796(31) \times 10^{-11} \mathrm{~m}^{3} /\left(\mathrm{kg} \mathrm{s}^{2}\right) .
$$

The CODATA recommended value of the Newtonian gravitational constant $G_{N}$ is equal to

$$
G_{N}=6.67408(31) \times 10^{-11} \mathrm{~m}^{3} /\left(\mathrm{kg} \mathrm{s}^{2}\right)[3] .
$$

The relative standard uncertainty of the gravitational constant $G$ in (15) is equal to $4.610^{-7}$ that is two order of magnitude less than relative standard uncertainty of the measured constant $G_{N}$, which is equal to $4.710^{-5}$ [3]. Thus, the accuracy of all the Planck units depending on the gravitational constant can be increased.

\section{Conclusions}

Thus, this work discovered a way to calculate the mass-spectrum of the charged leptons (the electron, muon, and tau) from the fundamental physical constants. The phenomenological formula includes the Planck mass as a scaling factor and dimensionless constants (the fine-structure constant is one of them). All the previous formulas considered the mass of charged leptons as fundamental constants between which there must be some connection.
This work tried to understand the origin of these particles.

\section{REFERENCES}

[1] P. Langacker. The Standard Model and Beyond (CRC Press, 2010).

[2] K. A. Olive, et al. (Particle Data Group). Review of particle physics. Chinese Physics C 38, 090001 (2014).

[3] P. J. Mohr, D. B. Newell, B. N. Taylor. CODATA Recommended Values of the Fundamental Physical Constants: 2014. Online available from http://arxiv.org/pdf/1507.07956.

[4] G. Preparata, S.-S. Xue. Do we live on a lattice? Fermion masses from the Planck mass. Phys. Lett. B 264, 35-38 (1991).

[5] Y. Nambu. An empirical mass spectrum of elementary particles. Prog. Theor. Phys. 7, 595-596 (1952).

[6] M. MacGregor. The Power of Alpha: The Electron Elementary Particle Generation with Alpha-Quantized Lifetimes and Masses (World Scientific Publishing, Singapore, 2007).

[7] A. O. Barut. Lepton mass formula. Phys. Rev. Lett. 42, 1251 (1979).

[8] A. O. Barut. [Erratum] Lepton mass formula. Phys. Rev. Lett. 43, 1057 (1979).

[9] R. Acharya, B. P. Nigam. Lepton-quark symmetry and Barut's lepton mass formula. Lettere al Nuovo Cimento (July 1981) 31, 437-438 (1981).

[10] A. Gsponer, J.-P. Hurni. Non-linear field theory for lepton and quark masses. Hadronic Journal 19, 367-373 (1996).

[11] Y. Koide. Fermion-boson two-body model of quarks and leptons and cabibbo mixing. Lettere al Nuovo Cimento 34, 201-205 (1982). 
[12] Y. Koide. A fermion-boson composite model of quarks and leptons. Phys. Lett. B 120, 161-165 (1983).

[13] C. G. Wohl, et al. (Particle Data Group). Review of particle properties. Rev. Mod. Phys. 56, S2-S304 (1984).

[14] Y. Sumino. Family gauge symmetry as an origin of Koide's mass formula and charged lepton spectrum. JHEP 0905, 075 (2009).

[15] E. M. Lipmanov. Phenomenology of the mass-spectrum of 6 generations of leptons. Soviet Journal of Nuclear Physics-USSR 40, 636-640 (1984).

[16] The ATLAS and CMS Collaborations. Combined Measurement of the Higgs Boson Mass in pp Collisions at $\sqrt{ }_{\mathrm{s}}=7$ and $8 \mathrm{TeV}$ with the ATLAS and CMS Experiments. Online available from http://arxiv.org/pdf/1503.07589.
[17] G. Cacciapaglia. The dynamics of Composite Higgses. Journal of Physics: Conference Series 623, 012006 (2015).

[18] P. G. Bergmann. The Riddle of Gravitation. (Scribner, New York, 1968).

[19] B. Bellazzini, C. Csáki, J. Serra. Composite Higgses. Eur. Phys. J. C 74, 2766 (2014).

[20] J. H. Conway, N. J. A. Sloane. Sphere Packing, Lattices and Groups (Springer-Verlag NY, 1998) - 3rd ed.

[21] O. R. Musin. The problem of the twenty-five spheres. Rus. Math. Surveys 58, 794-795 (2003).

[22] F. Pfender, G. M. Ziegler. Kissing Numbers, Sphere Packings, and Some Unexpected Proofs. Notices of the Am. Math. Soc. (September 2004), 873-883 (2004). 